\title{
Strong pulse asymmetry in quantum-dot mode-locked semiconductor lasers
}

Cite as: Appl. Phys. Lett. 98, 031104 (2011); https://doi.org/10.1063/1.3544579

Submitted: 05 November 2010 • Accepted: 31 December 2010 • Published Online: 20 January 2011

Mindaugas Radziunas, Andrei G. Vladimirov, Evgeny A. Viktorov, et al.

\section{ARTICLES YOU MAY BE INTERESTED IN}

Optimum phase noise reduction and repetition rate tuning in quantum-dot mode-locked lasers

Applied Physics Letters 104, 021112 (2014); https://doi.org/10.1063/1.4861604

Hybrid mode-locking in a $40 \mathrm{GHz}$ monolithic quantum dot laser

Applied Physics Letters 96, 011104 (2010); https://doi.org/10.1063/1.3279136

Stabilization of a passively mode-locked laser by continuous wave optical injection Applied Physics Letters 97, 101105 (2010); https://doi.org/10.1063/1.3483231

母 QBLOX

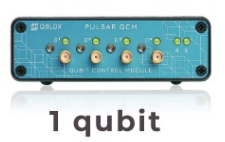

Shorten Setup Time Auto-Calibration More Qubits

Fully-integrated Quantum Control Stacks Ultrastable DC to $18.5 \mathrm{GHz}$ Synchronized $<1 \mathrm{~ns}$ ultralow noise

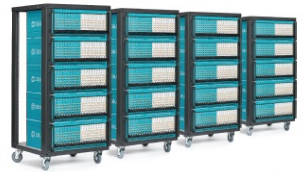

100s qubits

visit our website > 


\title{
Strong pulse asymmetry in quantum-dot mode-locked semiconductor lasers
}

\author{
Mindaugas Radziunas, ${ }^{1, a)}$ Andrei G. Vladimirov, ${ }^{1}$ Evgeny A. Viktorov, ${ }^{2}$ Gerrit Fiol, ${ }^{3}$ \\ Holger Schmeckebier, ${ }^{3}$ and Dieter Bimberg ${ }^{3}$ \\ ${ }^{1}$ Weierstrass Institute for Applied Analysis and Stochastics, Leibniz Institute, Forschungsverbund Berlin e. V. \\ Mohrenstrasse 39, 10117 Berlin, Germany \\ ${ }^{2}$ Optique Nonlinéaire Théorique, Université Libre de Bruxelles, Campus Plaine, CP 231, \\ B-1050 Bruxelles, Belgium \\ ${ }^{3}$ Institut für Festkörperphysik, Technische Universität Berlin, Hardenbergstr. 36, 10623 Berlin, Germany
}

(Received 5 November 2010; accepted 31 December 2010; published online 20 January 2011)

\begin{abstract}
We describe the formation of a strong pulse asymmetry in mode-locked quantum-dot edge-emitting two-section semiconductor lasers. A mode decomposition technique reveals the role of the superposition of different modal groups. The results of theoretical analysis are supported by the experimental data. (C) 2011 American Institute of Physics. [doi:10.1063/1.3544579]
\end{abstract}

The advantages of self-assembled quantum-dot (QD) materials ${ }^{1}$ can be exploited in multisection mode-locked (ML) lasers, which are able to generate stable high intensity picosecond and subpicosecond pulses. ${ }^{2}$ In this letter, we study experimentally and theoretically strongly asymmetric ML pulses generated by an edge-emitting QD ML laser consisting of a $100 \mu \mathrm{m}$ long saturable absorber (SA) and a $900 \mu \mathrm{m}$ long gain section. Our particular attention is drawn to the study of the pulses having a broad trailing edge plateau (TEP). ${ }^{3}$ Our theoretical analysis shows that the TEP in QD lasers arises mainly due to noninstant carrier transitions between the carrier reservoir (CR), excited state (ES), and ground state (GS) of the QDs. These multiple finite-time transitions slow down the carrier exchange between the electrically pumped CR and the photon generating GS of QD, act as a filtering, and lead to a homogenization of the carrier and photon distributions along the gain section. To reveal the role of separate optical modes in ML regimes with strongly asymmetric pulses, we have performed a modal analysis. ${ }^{4}$ The pulses with a strongly enhanced TEP can be represented as a superposition of a usual pulsating ML state formed by a large number of longitudinal modes and phase-shifted pulsations comprising only a few optical modes.

We consider a $1+1$ dimensional traveling wave model describing spatial-temporal evolution of the two counterpropagating optical fields, $E^{+}(z, t)$ and $E^{-}(z, t)$, material polarization functions, $p^{+}(z, t)$ and $p^{-}(z, t)$, normalized carrier density $n_{\mathrm{CR}}(z, t)$ within the CR, and occupation probabilities, $n_{\mathrm{GS}}(z, t)$ and $n_{\mathrm{ES}}(z, t)$, of the GS and ES of quantum dots, respectively. ${ }^{3}$

To describe carrier exchange between the CR, GS, and $\mathrm{ES}$ of the QDs in the SA $\left(z \in\left[0, l_{\mathrm{SA}}\right]\right)$ and gain $(z$ $\left.\in\left[l_{\mathrm{SA}}, L\right]\right)$ sections, we use the following rate equations: ${ }^{5}$

$$
\begin{aligned}
& \frac{d}{d t} n_{\mathrm{GS}}(z, t)=-\frac{n_{\mathrm{GS}}}{\tau_{\mathrm{GS}}}+2 R_{\mathrm{ES}, \mathrm{GS}}-\frac{1}{\Theta_{E}} R\left(n_{\mathrm{GS}}, E, p\right), \\
& \frac{d}{d t} n_{\mathrm{ES}}(z, t)=-\frac{n_{\mathrm{ES}}}{\tau_{\mathrm{ES}}}-R_{\mathrm{ES}, \mathrm{GS}}+R_{\mathrm{CR}, \mathrm{ES}},
\end{aligned}
$$

${ }^{a)}$ Electronic mail: radziuna@wias-berlin.de.

$$
\begin{aligned}
& \frac{d}{d t} n_{\mathrm{CR}}(z, t)=\frac{I(z)}{\theta_{I}}-\frac{n_{\mathrm{CR}}}{\tau_{\mathrm{CR}}}-4 R_{\mathrm{CR}, \mathrm{ES}}, \\
& R=\Re \mathrm{Re} \sum_{\nu= \pm} E^{\nu *}\left[2 g^{\prime}\left(n_{\mathrm{GS}}-\frac{1}{2}\right) E^{\nu}-\bar{g}\left(E^{\nu}-p^{\nu}\right)\right], \\
& R_{\mathrm{ES}, \mathrm{GS}}\left(n_{\mathrm{ES}}, n_{\mathrm{GS}}\right)=\frac{n_{\mathrm{ES}}\left(1-n_{\mathrm{GS}}\right)}{\tau_{\mathrm{ES} \rightarrow \mathrm{GS}}}-\frac{n_{\mathrm{GS}}\left(1-n_{\mathrm{ES}}\right)}{2 \tau_{\mathrm{GS} \rightarrow \mathrm{ES}}}, \\
& R_{\mathrm{CR}, \mathrm{ES}}\left(n_{\mathrm{CR}}, n_{\mathrm{ES}}\right)=\frac{n_{\mathrm{CR}}\left(1-n_{\mathrm{ES}}\right)}{4 \tau_{\mathrm{CR} \rightarrow \mathrm{ES}}}-\frac{n_{\mathrm{ES}}}{\tau_{\mathrm{ES} \rightarrow \mathrm{CR}}} .
\end{aligned}
$$

Here, the function $R$ stands for a stimulated recombination, $R_{\mathrm{ES}, \mathrm{GS}}$ and $R_{\mathrm{CR}, \mathrm{ES}}$ describe the carrier exchange rates between the dot's states, while $\tau_{a}^{-1}$ and $\tau_{a \rightarrow b}^{-1}, a, b$ $\in\{\mathrm{GS}, \mathrm{ES}, \mathrm{CR}\}$, denote spontaneous relaxation and transition rates between GS, ES, and CR, respectively. $\left(1-n_{\mathrm{GS}}\right)$ and $\left(1-n_{\mathrm{ES}}\right)$ represent the Pauli blocking, while factors 2 and 4 account for the degeneracy in the QD energy levels. Following Ref. 6, we assume that in the reversely biased SA section the carrier transitions from the CR to the ES can be neglected. Hence, in this section, we set $\tau_{\mathrm{CR} \rightarrow \mathrm{ES}}^{-1}=0$ and consider only the equations for $n_{\mathrm{GS}}$ and $n_{\mathrm{ES}}$ in Eq. (1).

The meaning and values of most of the laser parameters can be found in Ref. 3. In the present letter, we use the linewidth enhancement factor $\alpha_{H}=2$ and the scaling factors $\Theta_{I}=1.5 \mathrm{~A} \mathrm{ps}, \Theta_{E}=239 \mathrm{~W} \mathrm{ps} / \mathrm{m}$, which relate the gain section injection current $I_{G}=\left.I(z)\right|_{z \in\left[l_{S A}, L\right]}$ and the field intensity $\left|E^{ \pm}\right|^{2}$ with the scaling of $n_{\mathrm{CR}}$, the $\mathrm{QD}$ density, the electron charge, the group velocity, and the cross-sectional area of the active zone. In addition, in the gain section, we set $\tau_{\mathrm{ES}}$ $=\tau_{\mathrm{GS}}=1 \mathrm{~ns}, \quad \tau_{\mathrm{ES} \rightarrow \mathrm{GS}}=2 \mathrm{ps}, \quad \tau_{\mathrm{GS} \rightarrow \mathrm{ES}}=\tau_{\mathrm{CR} \rightarrow \mathrm{ES}}=5 \mathrm{ps}$, and $\tau_{\mathrm{ES} \rightarrow \mathrm{CR}}=80$ ps. Other parameters are the same as in Ref. 3 .

A single light pulse traveling back and forth the laser cavity and sequentially emitted at the SA and gain section facets [see functions $\left|E^{-}(0, t)\right|^{2}$ and $\left|E^{+}(L, t)\right|^{2}$ in Fig. 1(a)] corresponds to a fundamental ML regime. A fast increase of the carrier functions $n_{\mathrm{GS}}$ and $n_{\mathrm{ES}}$ in the SA section [see panel (c)] corresponds to a fast saturation of this section after the passage of a pulse. 


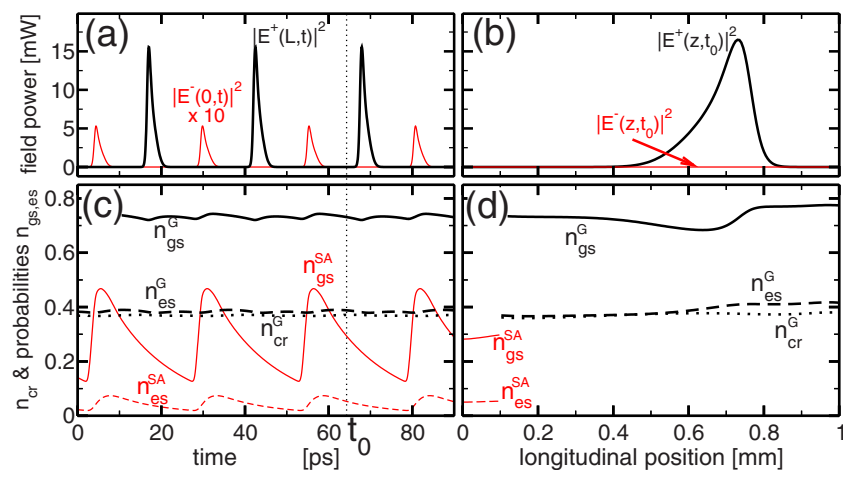

FIG. 1. (Color online) Typical fundamental ML pulsations $\left(U=-3 \mathrm{~V}, I_{G}\right.$ $=40 \mathrm{~mA}$ ). Left: time traces of the emitted field intensities (a) and the carrier functions $n_{\mathrm{GS}, \mathrm{ES}, \mathrm{CR}}$ averaged over the gain and SA sections (c), respectively. Right: axial distributions of the optical fields (b) and the carrier functions (d) at the time moment $t_{0}$.

Axial distributions of the intensities of two counterpropagating field amplitudes and carrier variables at the time moment $t_{0}$ are shown in panels (b) and (d) of Fig. 1. At this moment, the power of electromagnetic field is concentrated within a pulse traveling along the laser cavity in the forward direction [see the function $\left|E^{+}\left(z, t_{0}\right)\right|^{2}$ in Fig. 1(b)]. Panel (d) shows the depletion of the carriers in the gain section induced by this pulse. The strongest depletion corresponding to the largest pulse amplification is expected to take place twice per round trip when the pulse comes close to one of the two edges of the gain section. We note that in contrast to our previous study of a simplified two-carrier rate equation model, ${ }^{3}$ the depletion of $n_{\mathrm{CR}}(z)$ [panel (d)] as well as the oscillation amplitude of $n_{\mathrm{CR}}(t)$ [panel (c)] are much weaker. This is because the impact of the optical field onto the dynamics of the CR carrier density is additionally filtered by the intermediate rate equation for the ES occupation probability, which was omitted in our previous study. ${ }^{3}$

Figure 2 represents another typical ML regime computed with injection current $I_{G}$ twice as large as that used in Fig. 1. Let us emphasize the most important differences between these two ML regimes. (i) At larger injections, a ML pulse has a long TEP. (ii) While the emitted pulse energy increases with the injection current, the peak intensity of the output field remains almost unchanged. (iii) Carrier density $n_{\mathrm{CR}}$ of the gain section increases with the injection current. The occupation probabilities $n_{\mathrm{ES}}$ and $n_{\mathrm{GS}}$, which are pumped indirectly by $R_{\mathrm{CR}, \mathrm{ES}}$ and $R_{\mathrm{ES}, \mathrm{GS}}$, remain nearly unchanged. (iv) In panels (c) of both figures, the growth and decay rates of $n_{\mathrm{GS}}$

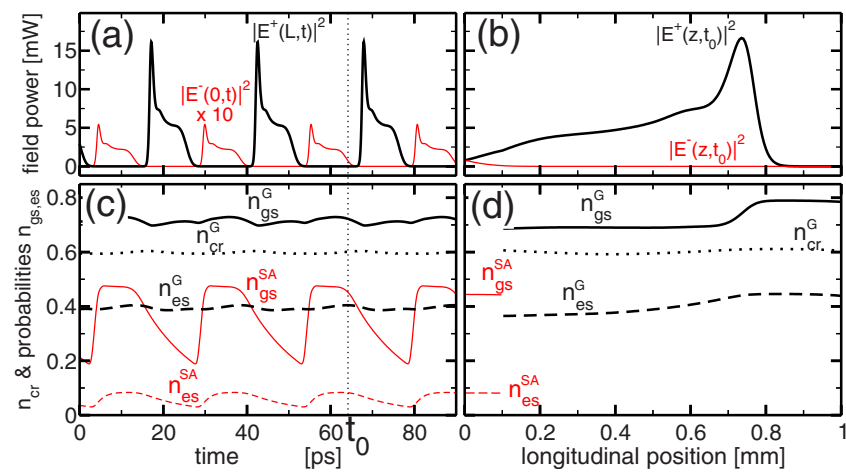

FIG. 2. (Color online) ML pulsation with a large plateau at the trailing edge of the pulse. The same as in Fig. 1, but for $I_{G}=80 \mathrm{~mA}$. in the SA section are similar. However, in Fig. 2, this probability has long flat maxima, indicating the absorber saturation by the long TEP of the ML pulse. These maxima correspond to the plateaus of pulses emitted at the SA facet [see solid gray curves in panels (c) and (a)]. The formation of strongly asymmetric pulses with TEP is unknown for the bulk or quantum well based devices, which directly confirms the role of at least one noninstant carrier transition between the CR and the GS.

In order to get a deeper understanding of the formation mechanisms of stable ML pulses with a long TEP, we have performed a modal analysis ${ }^{4}$ of the model equations. Introducing a differential operator $H(\beta)$, we rewrite the field equations ${ }^{3}$ in the operator form:

$$
\frac{d}{d t} \Psi(z, t)=H(\beta) \Psi(z, t), \quad \Psi=\left(E^{+}, E^{-}, p^{+}, p^{-}\right)^{T} .
$$

The field function $\Psi(z, t)$ can be decomposed into a series of instantaneous $[\beta(t)$-dependent $]$ modes,

$$
\Psi(z, t)=\sum_{k} f_{k}(t) \Theta_{k}(\beta, z) \Rightarrow E^{+}(L, t)=\sum_{k} f_{k}(t),
$$

where $\Theta_{k}$ and $f_{k}$ are properly scaled eigenfunctions of the spectral problem $H(\beta) \Theta(\beta, z)=i \Omega(\beta) \Theta(\beta, z)$ and complex modal functions representing the contribution of $k$ th mode to the field emitted from the gain section facet, respectively. $\Re$ e $\Omega_{k}$ and $\Im \mathrm{m} \Omega_{k}$ give a main contribution to the rotation and damping of the modal functions, ${ }^{4}$ which can be written in the form

$$
f_{k}(t)=f_{0}(t) s_{k}(t) e^{i k\left(2 \pi t / T_{c}-\psi_{k}(t)\right)}, \quad k \in \mathbf{Z} .
$$

Here, $s_{k}(t)=\left|f_{k} / f_{0}\right|$ and $\psi_{k}(t)$ are real slowly varying $T_{c}$-periodic functions, where $T_{c}$ is the period of ML regime close to the field round-trip time in the laser cavity. Relative phases $\psi_{k}(t)$ represent the phase difference between the $k$ th mode and the mode with the largest amplitude to which we assign the index " 0. ." The perfect locking between several equidistant modes having equal intensities corresponds to $\psi_{k}(t)=\bar{\psi}(t)$ and $s_{k}(t) \equiv 1$. Then the relation $t / T_{c}=\bar{\psi}(t) / 2 \pi \bmod (1)$ determines the time moments when the ML pulse peaks are expected.

The shape of a ML pulse depends strongly on the relations between the amplitudes and phases of the complex modal functions. Hence, using the mode decomposition (3), the contribution of the different modes into the formation of ML pulse can be revealed.

A total number of 200 modes centered around the gain peak frequency has been used in the decomposition of the field function $\Psi(z, t)$ (see Fig. 3). After that, a reconstruction of the electric fields has been performed with the help of a superposition of $m$ modes $(m=4,20,50)$ having largest amplitudes. We notice that the reconstruction with $m=50$ modes gives almost perfect approximation of the original field function, while 4- and 20-mode reconstructions are not sufficient to recover the precise shape of the calculated pulse.

The comparison of field reconstructions with different amount of modes shows an interesting feature of ML pulses with a broad TEP. For conventional ML state, an increase of the number of phase-locked modes usually leads to an increase of the pulse amplitude and a decrease of the pulse width without a significant shift of the pulse peak location. In 


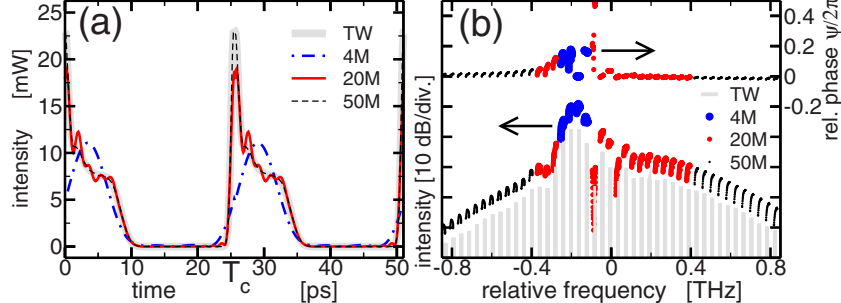

FIG. 3. (Color online) Modal analysis of a ML regime shown in Fig. 2. (a) Calculated time trace of the field intensity emitted at the gain section facet (thick gray curve) and its reconstruction using 50, 20, and 4 modes with largest $\left|f_{k}\right|$ (thin curves). (b) Bullets give a parametric representation of the modal phases $\psi_{k}(t)$ (above) and amplitudes $\left|f_{k}(t)\right|$ (below) vs modal frequencies $\Re$ e $\Omega_{k}(t)$. Optical spectrum of the emitted field is shown by the solid line. Bullets of different sizes indicate modes used for the field reconstruction in panel (a).

our case, however, when increasing the number $m$ from 4 to 50 , the peak location of the reconstructed pulse shifts by $15 \%-20 \%$ of the pulse repetition period $T_{c}$. This indicates that the strong asymmetry in the pulse shape appears not only due to larger amplitudes of a few most powerful central modes but also due to specific phase relations between these modes and the rest of the optical spectrum.

In Fig. 3(b), the amplitudes and relative phases of modes are shown versus their frequency. All these modes give a similar contribution to the formation of the sharp main peaks of the ML pulses located at $t \approx 0$ and $t \approx T_{c}$ in Fig. 3(a). Only a few most powerful modes in the central part of the optical spectrum have the phases $\psi_{k}$ shifted from zero by approximately $15 \%-20 \%$ of the period. These shifts correspond to the shift of the TEP from the position of the main peak [see the dashed-dotted line in Fig. 3(a)]. Therefore, we can conclude that TEP is formed by a few most powerful modes.

Thus, an optical field of a ML pulse with a TEP can be represented as a sum of two components: $E^{+}(L, t)=E_{1}(t)$ $+E_{2}(t)$. The intensities of the components, $\left|E_{1}(t)\right|^{2}$ and $\left|E_{2}(t)\right|^{2}$, have the same periodicity $T_{c}$. While $\left|E_{1}(t)\right|^{2}$ has a form of a narrow high intensity pulse, $\left|E_{2}(t)\right|^{2}$ corresponds to a rather broad pulse with a time-shifted peak and a smaller peak power.

Experimental studies of the ML pulse broadening have been performed with a ridge waveguide two-section QD monolithic ML laser. The material incorporated is InGaAs, forming 15 stacked layers of QDs. The device was integrated in a module comprising a fiber pigtail, a microwave port, dc contacts, and a thermoelectric cooler. ${ }^{7,8}$

Figure 4 gives two different time-domain representations of the pulses measured for several injection currents $I_{G}$. The autocorrelation (AC) functions of these pulses [panel (a)] clearly show a typical increase of the pulse width with injection current. Note that for $I_{G}=100 \mathrm{~mA}$, the AC function has nonvanishing wings, which is typical for pulses with the intensity remaining distinguishable from zero for more than half of the pulse repetition period.

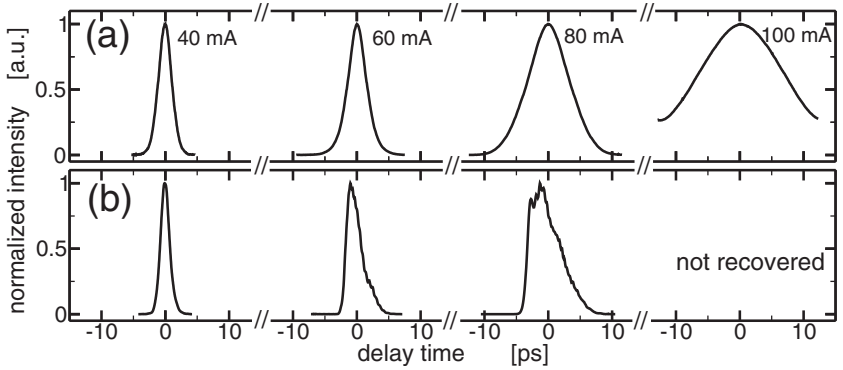

FIG. 4. Experimental AC measurements (a) and reconstructed pulse shapes using FROG technique (b) at fixed voltage of the SA section and several values of the injection currents into the gain section.

Figure 4(b) shows the corresponding pulses reconstructed by means of the frequency-resolved optical gating (FROG) technique. ${ }^{9}$ Similar to our theoretic predictions, the shape of the front edge of the pulse remains nearly independent of $I_{G}$, while the pulse broadening is due to the growing trailing edge. At higher currents $\left(I_{G}=80 \mathrm{~mA}\right)$, the formation of the TEP is visible. At even higher injections, however, the pulse reconstruction fails since the FROG algorithm requires zero intensity at the edges of the corresponding $\mathrm{AC}$ functions.

In conclusion, we demonstrate numerically and experimentally that the increase of the injection current in a monolithic two-section mode-locked QD laser leads to the formation of ML pulses with a large TEP formed by a few central modes having much larger amplitudes than the remaining ML modes.

The work of M. Radziunas was supported by DFG Research Center MATHEON. A. G. Vladimirov and the authors from TU Berlin (G. Fiol, H. Schmeckebier, and D. Bimberg) acknowledge the support from SFB 787 project of the DFG. E. A. Viktorov is thankful to the support of Fond National de la Recherche Scientifique (Belgium).

${ }^{1}$ N. N. Ledentsov, D. Bimberg, and Z. I. Alferov, J. Lightwave Technol. 26, 1540 (2008).

${ }^{2}$ E. U. Rafailov, M. A. Cataluna, and W. Sibbett, Nat. Photonics 1, 395 (2007).

${ }^{3}$ M. Radziunas, A. G. Vladimirov, and E. A. Viktorov, Proc. SPIE 7720, $77200 X(2010)$.

${ }^{4}$ M. Radziunas and H.-J. Wünsche, Optoelectronic Devices-Advanced Simulation and Analysis, edited by J. Piprek (Springer, New York, 2005), Chap. 5, pp. 121-150.

${ }^{5}$ A. Markus, M. Rossetti, V. Calligari, D. Chek-Al-Kar, J. X. Chen, and A. Fiore, J. Appl. Phys. 100, 113104 (2006).

${ }^{6}$ E. A. Viktorov, T. Erneux, P. Mandel, T. Piwonski, G. Madden, J. Pulka, G. Huyet, and J. Houlihan, Appl. Phys. Lett. 94, 263502 (2009).

${ }^{7}$ G. Fiol, C. Meuer, H. Schmeckebier, D. Arsenijevic, S. Liebich, M. Laemmlin, M. Kuntz, and D. Bimberg, IEEE J. Quantum Electron. 45, 1429 (2009).

${ }^{8}$ H. Schmeckebier, G. Fiol, C. Meuer, D. Arsenijevic, and D. Bimberg, Opt. Express 18, 3415 (2010).

${ }^{9}$ D. J. Kane and R. Trebino, IEEE J. Quantum Electron. 29, 571 (1993). 